\title{
Intelligent Nebulizers in the Age of the Internet: The I-neb Adaptive Aerosol Delivery (AAD) System
}

\author{
Rajiv Dhand, M.D., FCCP
}

$\mathbf{T}$ He I-neb Adaptive Aerosol Delivery (AAD) System is among the most technologically advanced and sophisticated nebulizer systems in clinical use. In the United States, the I-neb AAD System is approved for the delivery of inhaled iloprost, whereas in Europe it is approved as a multipurpose nebulizer that is employed for specific applications where it is included in the drug license. This special supplement is sponsored by Philips Respironics (UK) Ltd, and contains eight articles that comprehensively review technical details of the I-neb AAD System and provide data on patient use in domiciliary settings. ${ }^{(1-8)}$ This issue, focused exclusively on a single aerosol delivery device, is a unique publication for the Journal. Hopefully, this will be a harbinger of other supplements to follow so that the medical community may learn detailed and peerreviewed information about newer aerosol delivery devices.

Conventional nebulizers have several well-recognized drawbacks that make them unsuitable for administration of therapy with expensive medications, especially those that require precise dosing. ${ }^{(9)}$ The unique features of the I-neb AAD System overcome many shortcomings of conventional nebulizers. $^{(1)}$ Some of the distinctive features of the I-neb AAD System are its ability to provide: (1) precise dosing, (2) feedback to the patient, (3) ability to log information about device use and performance, and (4) data for transmission over the Internet to remotely monitor patient's adherence to the drug regimen and device performance. $(1,3)$

\section{Precision of Dosing}

The I-neb AAD System, the latest generation of "intelligent" nebulizers, is based on a vibrating mesh nebulizer platform coupled with AAD technology. ${ }^{(1,2)}$ Software in the I-neb AAD System analyzes the patient's breathing pattern and pulses aerosol only during inspiration, thereby avoiding aerosol waste during expiration. Precise dosing by the I-neb AAD System is facilitated by a unique metering chamber design. ${ }^{(2)}$ Similar to other vibrating mesh nebulizers, ${ }_{(1,2)}^{(10}$ the I-neb AAD System has a minimal residual volume. ${ }^{(1,2)}$ The metered dose of the drug can be adjusted based on the nominal dose placed in the metering chamber, the residual volume, drug deposition in the mouthpiece, and the small proportion of exhaled drug $(<1 \%)$. ${ }^{(2,4)}$

The majority of drug in the aerosol (60 to $80 \%$ ) is contained within the fine particle fraction (mass in particles with mass median diameter $<5 \mu \mathrm{m} /$ emitted dose). ${ }^{(2)}$ The metered dose is within $25 \%$ of the nominal dose, and the dose delivered to the patient is within $10 \%$ of the expected metered dose. ${ }^{(2,11)}$ Because the I-neb AAD System takes into account the breathing pattern of the individual patient, variations in delivered dose due to the patient's breathing pattern are minimized. Thus, the dose delivered to the lungs can be predicted with reasonable accuracy by in vitro tests. $(2,4)$

Due to the higher efficiency of the I-neb AAD System, when transitioning from conventional nebulizers it is important to note that a threefold reduction in drug volume and up to fivefold reduction in nominal dose may be needed with the I-neb AAD System to achieve similar amounts of lung deposition. ${ }^{(2)}$ Likewise, a previous study by our group found that a similar bronchodilator response could be achieved in patients with chronic obstructive pulmonary disease (COPD) with 2.5- to fivefold lower nominal dose of ipratropium bromide delivered with a vibrating mesh nebulizer compared to a conventional jet nebulizer. ${ }^{(12)}$

Aerosol delivery with the I-neb AAD System is guided by two algorithms: one for the Tidal Breathing Mode (TBM) and one for slow and deep inhalations, the Target Inhalation Mode (TIM). These modes are described in detail in this supplement. The TBM is suitable for most adults and for children older than 2 years of age. ${ }^{(10)}$ The TIM is a unique feature of the I-neb AAD System, ${ }^{(1,2,4-7)}$ but is not yet commercially available in the United States. For optimal use of the TIM, patients need to have a forced vital capacity $\geq 1.75 \mathrm{~L}$. ${ }^{(5)}$ Thus, the TIM could be challenging for small children, patients with poor lung capacity, and patients who are hyperventilating or tachypneic due to acute or chronic illness.

The advantages of a slow and deep inhalation in terms of increased pulmonary drug deposition are well known. ${ }^{(13-15)}$ Mean lung deposition of radiolabeled aerosol $\left({ }^{99 \mathrm{~m}} \mathrm{Tc}-\mathrm{DTPA}\right.$ in normal saline) with the I-neb AAD System was $62.8 \%$ in TBM and $73.3 \%$ in TIM. ${ }^{(4)}$ Breathing with the TIM also reduces total treatment time. ${ }^{(4,6,7)}$ The mean treatment time in patients with cystic fibrosis decreased from $6.83 \mathrm{~min}$ with TBM to $4.2 \mathrm{~min}$ with TIM, and the time for each treatment decreased as the duration of inspiration in the TIM was increased over several days of use. ${ }^{(6)}$ At first glance, this may appear to be paradoxical because patients breathe at a slower rate during TIM than TBM; however, the total inspiratory time/min is considerably longer during TIM than TBM. 
Because the I-neb AAD System delivers aerosol only during inspiration, the time for aerosol delivery $/ \mathrm{min}$ is increased during TIM compared to TBM. ${ }^{(6)}$

\section{Feedback to the Patient}

With the introduction of the hydrofluoroalkane propelled pressurized metered-dose inhalers (HFA-pMDIs) and as previously noted with some dry powder inhalers (e.g., Turbuhaler does not employ large carrier particles, such as lactose, for drug delivery), patients are often unsure about whether they have actually received a dose from their inhalation device. To overcome this difficulty, the I-neb AAD System provides primary feedback to the patient in the form of a smiling face on the LCD screen (visual), a buzzer (audible), and vibratory (tactile) signals when nebulization of the dose preprogrammed on the AAD Disc is completed. ${ }^{(1)}$ The I-neb AAD System also provides a secondary feedback on each inhalation. During TBM, a vibration signals the start of aerosol delivery, whereas in TIM the vibration occurs at the end of each inhalation when the patient reaches the target inhalation time. Most patients find this feedback reassuring and "pleasant."(5,6) The system allows the patient to take pauses during treatment. These features account for satisfactory conclusion of more than $80 \%$ of the treatments with the I-neb AAD System. ${ }^{(6)}$

\section{Patient Logging System}

Accurate monitoring of device use is a commonly encountered clinical problem with several aerosol delivery devices. For pMDIs, until recently there was no counter to keep track of the number of doses remaining in the device, and this could lead to problems during an acute exacerbation if there was insufficient drug remaining in the pMDI. ${ }^{(16)}$ In the I-neb AAD System, a patient logging system (PLS), consisting of a memory chip and an infrared interface to transmit the data to a computer, allows monitoring of patient adherence. The microchip of the AAD Disc is different for each drug formulation, is preprogrammed for each drug, records details of the treatments, and counts down the number of remaining doses. No medication is provided once the specified numbers of doses have been used.

The PLS data is accessed via the I-neb Insight function, a PC-based software program with an infrared interface. These logged data can be used to monitor long-term patient adherence and device performance. The interactive screen display can also be employed to train patients for the slow and deep inhalations needed for the TIM.

\section{Telehealth Capability}

The potential to monitor and manage the patient's inhalation therapy by remote access is another innovative feature of the I-neb AAD System. ${ }^{(3)}$ Data from the AAD Disc can be downloaded via a modem to a monitoring station. These data are reviewed and an electronic prescription request generated for the pharmacy to provide additional drug. The pharmacy then makes arrangements to deliver the prescription to the patient's home. This program has been shown to be feasible and acceptable to patients. ${ }^{(3)}$ As the Internetbased technology continues to evolve, clinicians will have an unprecedented access to data that allows them to remotely monitor the patient's adherence to treatment, as well as to identify problems with the usage of the I-neb AAD System and any side effects of the treatment on a day-to-day basis. Electronic prescriptions and home delivery of medications could reduce the number of clinic visits and facilitate management of patients requiring many inhaled therapies on a daily basis.

Similar to other aerosol delivery devices, the performance of the I-neb AAD System in clinical use is critically dependent on following the manufacturers' instructions. The instructions for use are individualized based on the drug being nebulized, and the guidelines for use vary in different countries depending on local clinical practice and regulatory approval. A general guide to clinicians and patients is available at http://ineb.respironics.com/elearning.asp, but may need to be adapted according to the factors mentioned above. Patients need to be trained in the appropriate use of the I-neb AAD System at the time when they first use the device and during follow up clinic visits.

\section{Advantages of the I-neb AAD System}

The most attractive feature of the I-neb AAD System is that there is a potential for a single platform to deliver multiple inhaled drug therapies, such as those employed by patients with cystic fibrosis (bronchodilators, corticosteroids, mucolytics, hypertonic saline, and antibiotics). The I-neb AAD System contains a rechargeable battery, and it is a portable system that provides noise-free operation, low residual volume, and high fine particle fraction in the aerosol produced along with the ability to adapt to the patient's breathing pattern. Synchronizing aerosol delivery with a portion of the inspiration minimizes drug exposure for parents and caregivers. Because the formulations do not contain propellants the I-neb AAD System is environmentally friendly. The ability to count doses and monitor patient adherence and device performance are other obvious advantages. Patients also appreciate the training on using the device and various feedback signals it provides to guide treatment. These features are associated with a high level of patient acceptance and satisfaction with the I-neb AAD System. ${ }^{(6,8)}$ The capability to remotely manage the patient's inhalation therapy is exciting, ${ }^{(17)}$ but its full impact needs further investigation.

\section{Disadvantages of the I-neb AAD System}

The high cost of the I-neb AAD System is an obvious drawback. Whether the device provides added value in terms of patient acceptance, adherence, and improvement in outcomes has not been well established as yet. The I-neb AAD System has been recently introduced, and is only approved in the United States for inhalation of iloprost in patients with pulmonary hypertension. Thus, many patients and clinicians lack familiarity with the I-neb AAD System and may not be able to provide accurate information about its proper use. Practitioners will need to be specially trained to provide adequate instruction to patients. The device must be thoroughly cleaned immediately after each use to prevent clogging of the apertures in the vibrating mesh. The appropriate method of cleaning was, unfortunately, not adequately addressed by the articles in this Supplement. Likewise, more information is needed about how frequently the device or the mesh has to be 
replaced, and how long it will maintain its performance in "real-world" settings. The I-neb AAD System was shown to be efficient for delivering several drugs ${ }^{(2)}$ and $\alpha 1$ antitrypsin, ${ }^{(7)}$ but further experience is needed with a variety of drugs and biological agents. Although the I-neb AAD System is smaller and lighter than previous AAD devices, ${ }^{(1)}$ further reductions in size would enhance its appeal and portability. The I-neb AAD System may not be suitable for all populations, especially children $<2$ years of age, the mentally handicapped, and the very old. Poor lung capacity and rapid respiratory rates could be impediments to effective use of the TIM mode. The I-neb AAD System has not yet been adapted for use in mechanically ventilated patients. Furthermore, the need to use different AAD Discs for each formulation could be confusing for patients using multiple inhaled drugs, especially if it is also required to change mouthpieces while switching from the TBM to the TIM mode. Drugs administered by the I-neb AAD System cannot be mixed together; therefore, significant amounts of time would be needed to complete administration of multiple drugs. Because many patients who utilize nebulizer therapy are chronically ill and have a limited functional reserve, taking prolonged inhalations over several minutes could be tiring for such patients.

In the age of the Internet, people have enthusiastically embraced iPhones and iPods ${ }^{\circledR}$ into their daily lives. The I-neb AAD System is, therefore, very much in synch with the times. The obvious question is whether the I-neb AAD System is a sophisticated gadget or an important clinically relevant advance in inhalation therapy. How useful will this device be in the clinician's armamentarium, and will it have a significant impact on patient outcomes? How does the I-neb AAD System measure up to the requirements of an ideal aerosol delivery device, and in what clinical circumstances would it be a "natural" choice for administering inhaled therapy? Further studies and clinical experience will provide answers to these important questions. In the meantime, we can take comfort in the knowledge that the science of nebulization has taken a significant leap forward with the development of the I-neb AAD System.

\section{Author Disclosure Statement}

The author has received speaker fees from GSK and Boehringer Ingelheim, and consulting fees from Bayer. He has also received funding for participating in clinical trials from GSK and Novartis.

\section{References}

1. Denyer J, and Dyche T: The Adaptive Aerosol Delivery (AAD) technology: past, present and future. J Aerosol Med Pulm Drug Del. 2010;23(Suppl 1):S-1-S-10.

2. Hardaker LEA, and Hatley RHM: In vitro characterization of the I-neb Adaptive Aerosol Delivery (AAD) System. J Aerosol Med Pulm Drug Del. 2010;23(Suppl 1):S-11-S-20.

3. Nikander K, Denyer J, Dodd M, Dyche T, Webb K, Weller P, and Stableforth D: The Adaptive Aerosol Delivery System in a telehealth setting: patient acceptance, performance, and feasibility. J Aerosol Med Pulm Drug Del. 2010;23(Suppl 1): S-21-S-27.

4. Nikander K, Prince I, Coughlin S, Warren S, and Taylor G: Mode of breathing-Tidal or slow and deep-through the I-neb Adaptive Aerosol Delivery (AAD) system affects lung deposition of ${ }^{99 \mathrm{~m}}$ Tc-DTPA. J Aerosol Med Pulm Drug Del. 2010;23(Suppl 1):S-37-S-43.

5. Denyer J, Prince I, Dixon E, Agent P, Pryor J, and Hodson M: Evaluation of the Target Inhalation Mode (TIM) breathing maneuver in simulated nebulizer therapy in patients with cystic fibrosis. J Aerosol Med Pulm Drug Del. 2010;23(Suppl 1): S-29-S-36.

6. Denyer J, Black A, Nikander K, Dyche T, and Prince I: Domiciliary experience of the Target Inhalation Mode (TIM) breathing maneuver in patients with cystic fibrosis. J Aerosol Med Pulm Drug Del. 2010;23(Suppl 1):S-45-S-54.

7. Geller DE, and Kesser KC: The I-neb Adaptive Aerosol Delivery System enhances aerosol delivery of $\alpha 1$-antitrypsin with controlled inhalation. J Aerosol Med Pulm Drug Del. 2010;23(Suppl 1):S-55-S-59.

8. Goodman N, Morgan M, Nikander K, Hinch S, and Coughlin S: Evaluation of patient-reported outcomes and quality of life with the I-neb AAD system in patients with chronic obstructive pulmonary disease. J Aerosol Med Pulm Drug Del. 2010;23(Suppl 1):S-61-S-70.

9. Rau JL: Design principles of liquid nebulization devices currently in use. Respir Care. 2002;47:1257-1275.

10. Dhand R: Nebulizers that use a vibrating mesh or plate with multiple apertures to generate aerosol. Respir Care. 2002;47: 1406-1416.

11. Van Dyke R, and Nikander K: Delivery of iloprost inhalation solution with HaloLite, Prodose, and I-neb Adaptive Aerosol Delivery (AAD) systems - an in vitro study. Respir Care. 2007;53:184-190.

12. Dhand R, Giri VV, Noth I, Lissin D, Fishman RS, and Taylor $\mathrm{K}$ : Bronchodilator response to ipratropium delivered via Aerodose inhaler or Pari LC Plus nebulizer in COPD. Am J Respir Crit Care Med. 2002;165:A593.

13. Newman SP, Pavia D, Garland N, and Clarke SW: Effects of various inhalation modes on the deposition of radioactive pressurized aerosols. Eur J Respir Dis. 1982;63(Suppl):57-65.

14. Bennett WD, and Smaldone GC: Human variation in the peripheral air-space deposition of inhaled particles. J Appl Physiol. 1987;62:1603-1610.

15. Brand P, Schulte M, Wencker M, Herpich CH, Klein G, Hanna K, and Meyer T: Lung deposition of inhaled alpha 1proteinase inhibitor in cystic fibrosis and alpha1-antitrypsin deficiency. Eur Respir J. 2009;34:354-360.

16. Sander N, Fusco-Walkert SJ, Harder JM, and Chipps BE: Dose counting and the use of pressurized metered-dose inhalers: running on empty. J Allergy Asthma Immunol. 2006; 97:34-38.

17. McNamara P, McCormack P, McDonald AJ, Heaf L, and Southern KW: Open adherence monitoring using data download from an adaptive aerosol delivery nebulizer in children with cystic fibrosis. J Cystic Fibrosis. 2009;8:258-263.

Rajiv Dhand, M.D., FCCP Professor of Medicine Division Director

Division of Pulmonary, Critical Care and Environmental Medicine University of Missouri Staff Physician

Harry S. Truman VA Hospital

Columbia, $\mathrm{MO}$

E-mail: dhandr@health.missouri.edu 